\title{
Scaling of the urban heat island effect based on the energy balance: nighttime minimum temperature increase vs. urban area length scale
}

\author{
T. W. Lee* ${ }^{*}$ A. Ho \\ Department of Mechanical and Aerospace Engineering, Arizona State University, Tempe, Arizona, USA
}

\begin{abstract}
One of the adverse results of urbanization is the urban heat island (UHI) effect, wherein a combination of various factors renders the temperatures in an urban region higher than in the surroundings. We examined the UHI effect in 2 cities: Phoenix and Tucson (Arizona, USA), with disparate length scales but similar meteorological conditions. Based on analyses of both the urbanized land surface areal growth and thermal energy balance, a correlation appears wherein the nighttime minimum temperature (an indicator of the UHI effect) is seen to follow the square-root of the area, or the length scale of the urbanized land area. Using thermal energy transport for the air mass above the urbanized area, this scaling can be explained by a time-dependent energy balance, which shows that the rate of decrease in temperature also scales linearly with the length scale of the urban area. Viewed in this perspective, the length scale of an urbanized area is an important parameter in determining the severity of the UHI effects, as the advection rate is diminished when the length scale of the urban area is increased. Based on this scaling, the UHI effect, as indicated by nighttime temperature deviations from undisturbed levels, will tend to increase as the square-root of the urbanized area due to the advection rate effect.
\end{abstract}

KEY WORDS: Urban heat island · Scaling · Energy balance · Urbanized land surface area

\section{INTRODUCTION}

The worldwide trend of movement of human populations toward urban centers makes it imperative that a stable urban environment is sustained for the present and the future. One of the adverse effects of urbanization is the urban heat island (UHI) effect wherein a combination of various anthropogenic activities renders the temperatures in an urban area higher than that in the surrounding, nondeveloped areas. There have been many studies on the causes and impacts of the UHI effect (e.g. Rydin 1992, Pearlmutter \& Berliner 1998, Brazel et al. 2000, Stone \& Rogers 2001, Baker et al. 2002, Kalnay \& Cai 2003, Christy et al. 2006, De Laat \& Maurellis 2006). A wide range of causes have been implicated, including land surface energy balance (or imbalance) due to urbanized land surface and built structures, anthropogenic heat release and dif- ferent atmospheric constituents over the city (Landsberg 1979).

Kalnay \& Cai (2003) reported in a large-scale study on the effect of land use on temperature bias, and found a large increase in the nighttime minimum temperature with urbanization of the land in the continental USA. Several other studies point to a correlation between the urban UHI effect and the urbanized land surface. For example, Gallo et al. (1995) used satellite image data to obtain the normalized vegetation index in 28 cities, and found this index to be directly correlated with the increase in the nighttime minimum temperatures, i.e. when the vegetation index decreases, the nighttime minimum temperature increases. A study by Chen et al. (2006) reported on a similar correlation between the urban land area and nighttime minimum temperature in Shenzhen, China. Several other studies confirm the relationship between the urban 
area and the UHI intensity either directly or indirectly (Landsberg 1975, Yague et al. 1991, Bacci \& Maugeri 1992, Chow 1992). On the other hand, numerical simulations by Atkinson (2003) comparing various effects on UHI, showed that the size of the urban area had a minimal effect on the UHI intensity; instead, anthropogenic heat release had a significant effect. This is similar to the argument of Oke (1973) wherein a functional relationship was shown between UHI intensity and the urban population. Park (1986) showed that UHI intensity increases with both the population and the urban area ('impermeable area'), and decreases with wind speed. The latter point is quite important since wind speed is a measure of the effectiveness of advective heat removal; if wind speed is an important factor, then the length scale over which the advection effect takes place, i.e. the length scale of the urban area, should also be important.

We studied 2 of the warmest urban areas in the USA, the Phoenix and Tucson metropolises, and the relationship between the measured temperatures and the change in the urban land surface area. Phoenix is a rapidly growing city in terms of both land coverage and population, and exhibits one of the highest rates of increase in nighttime minimum temperature. Phoenix has been the subject of continuing UHI studies (Stabler et al. 2005, Brazel et al. 2007, Emmanuel \& Fernando 2007, Di Sabatino et al. 2009). Stabler et al. (2005), for example, found that the Phoenix UHI is directly correlated with the growth of the urban land area. A significant effect of the surface (e.g. urbanized, agricultural) was observed in a comprehensive study of the Phoenix UHI by Brazel et al. (2007). The 2 metropolitan areas of Phoenix and Tucson have disparate scales (by a factor of $\sim 5$ ) in terms of the urbanized surface, and yet have similar meteorological boundary conditions in terms of annual temperature ranges, precipitation and surface albedo. City structures in both Phoenix and Tucson are uniform in their height to width ratios in that high-rise buildings are small in number and concentration. Moreover, similar materials are used in pavements and building facades. This constitutes a rare opportunity to compare the 2 urban areas and isolate the effects of the urbanized land area.

We used satellite image data to observe the changes in the urbanized land area of Phoenix and Tucson, and establish a relationship with the nighttime minimum temperatures. We used a theoretical model based on a time-dependent energy balance over urbanized surfaces, to present a thermo-physical mechanism and a mathematical relationship between the urbanized land length scale and UHI intensity, the latter being observed as an increase in nighttime minimum temperatures.

\section{METHODS}

Subsets of Landsat Thematic Mapper (TM) data were analyzed for identification of the urbanized land surface and its area in the Phoenix and Tucson metropolitan areas. The Landsat TM is a comprehensive earth-observation image data set encompassing 7 spectral bands with $30 \mathrm{~m}^{2}$ spatial resolution, except for the thermal band. With a swath width of $185 \mathrm{~km}$, a region is observed every $16 \mathrm{~d}$. The selection of the analysis dates for a given year was variable, depending on the quality of the images and availability of auxiliary data (aerial image for validation and correction of the classification algorithm, see below). We focused on obtaining optimal data, as opposed to sampling at fixed intervals, since the urbanized land surface area did not change appreciably within a year and we were primarily interested in the trends in the data. For the Phoenix metropolitan area, the subset covered a region of $120 \times 90 \mathrm{~km}$ with $4000 \times 3000$ pixels, while the Tucson coverage was $60 \times 60 \mathrm{~km}(2000$ $\times 2000$ pixels). In both cases, the subsets were more than sufficient to completely envelop the metropolitan areas. Because the total urbanized area of the cities is the only quantity of interest, it was expedient to perform a binary classification to separate developed areas from other classes of land (e.g. undisturbed, vacant, water, and vegetation). Assigning a value of ' 1 ' to designate urbanized areas and a value of ' 0 ' for all other areas, a multiple linear regression was carried out, with the values in the 7 bands of the Landsat TM data as predictor variables. Some of the details of the land surface classification are described below, and further details are available in Ho (2005).

The classification algorithm developed has the following form:

Land cover $=1$, if $0.01868 \bar{b}_{1}-0.0095 \bar{b}_{5}>0.847$ and $\bar{b}_{1}>100$

$$
=0 \text {, otherwise }
$$

The quantities $\bar{b}_{1}$ and $\bar{b}_{5}$, respectively represent the standardized Landsat TM Band 1 (0.45 to $0.52 \mu \mathrm{m})$ and Band $5(1.55$ to $1.75 \mu \mathrm{m})$ intensity values. Although the above classification algorithm was reasonably accurate for most land surfaces, there were ambiguities for the mountainous areas and certain riverbeds that were improperly classified as urbanized. For this reason, the results were manually corrected by observing a $500 \times$ 500 pixel $(15 \times 15 \mathrm{~km})$ window and determining the proportion of misclassified pixels in the window, and subtracting the total number of misclassified pixels in all windows from the original estimate. Mathematically, Eq. (2) describes the correction procedure as :

$$
N^{*}=\sum_{i=1}^{m} n_{i}\left(1-p_{i}\right)
$$


where $N^{*}$ represents the revised estimate of urbanized pixels in a total scene, $i$ is the sub-image identifier, $m$ is the total number of sub-images ( $m=48$ and 20 for the Phoenix and Tucson images, respectively), $n_{i}$ is the uncorrected estimate of urbanized pixels in the $500 \times$ 500 pixel window, and $p_{i}$ is the estimated proportion of misclassified pixels in the window.

The proportion of misclassified pixels $p_{i}$ was determined by inspecting each sub-image and comparing it with aerial images with $1 \mathrm{~m}$ spatial resolution to identify the misclassified urban pixels. Because the algorithm usually resulted in a false positive when there was an error (i.e. pixels were misclassified as urbanized when they were not), a simplifying assumption was that all pixels initially classified as undeveloped were truly undeveloped, eliminating the need to check those pixels. The proportion $p_{i}$ was determined through inspection of questionable pixels that were initially classified as urbanized. By comparing these positively classified pixels to aerial photographs taken between 1992 and 1997 (spatial resolution of $1 \mathrm{~m}$ ), it was possible to reasonably determine whether the questionable pixels were misclassified. The time period when aerial imagery was available fell within the time interval when the Landsat TM images were acquired, but the number of questionable pixels was generally small ( 8\%). Nevertheless, the uniform application of criteria to these pixels provides a method of evaluating the accuracy of the classification method.

The temperature data were obtained from the Global Historical Climate Network (GHCN). The details of the temperature records can be found in the GHCN website (http://cdiac.esd.ornl.gov/ghcn/ghcn. html). In the metropolitan Phoenix area, there are $>12$ substations where temperature records are taken. The nighttime minimum temperature data follow consistently similar trends among substations, from Phoenix,

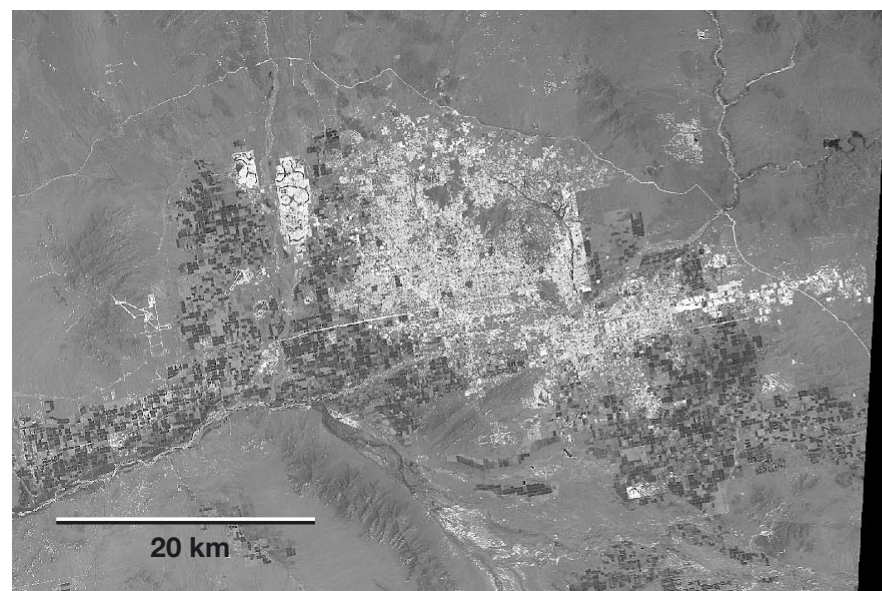

Phoenix Sky Harbor Airport, Tempe and Mesa (see Fig. 4d in Brazel et al. 2000), with central locations exhibiting slightly higher temperatures than outlying areas. We used the average of the 3 monthly averaged temperatures (for June, July and August) at the Phoenix Sky Harbor International Airport site due to its central location in the Phoenix metropolitan area and the representative nature of the temperature data at this site.

\section{RESULTS AND DISCUSSION}

\subsection{Landsat image analysis}

Figs. $1 \& 2$ show the LANDSAT images of the Phoenix and Tucson areas taken in 1984/1985 and 1998. There has been a substantial increase in the urbanized land area for Phoenix, with spreading occurring in all of the peripheral regions of the city. Dendritic growths also tend to merge to form continuous sections of urbanized land. For the Tucson area, growth is not as dramatic, with most of the urbanization occurring northwest of the city boundary. The population densities in Phoenix and Tucson are similar, at 2700 ind. mile $^{-2}$ (Rex 2000). Although there is the usual variation of high to low population densities as one moves from the urban center toward the periphery, the changes in the local population density have been very small and population density can be considered as a constant. Based on these images in Figs. 1 \& 2, the image analysis algorithm discussed in the previous section gives a quantitative measure of the increase in the urbanized land area for both metropolitan areas as a function of time. Although the classification algorithm uses bands 1 and 5 of the Landsat data set, the black and white images are used in Figs. $1 \& 2$ to qualitatively show the urban areal growth.

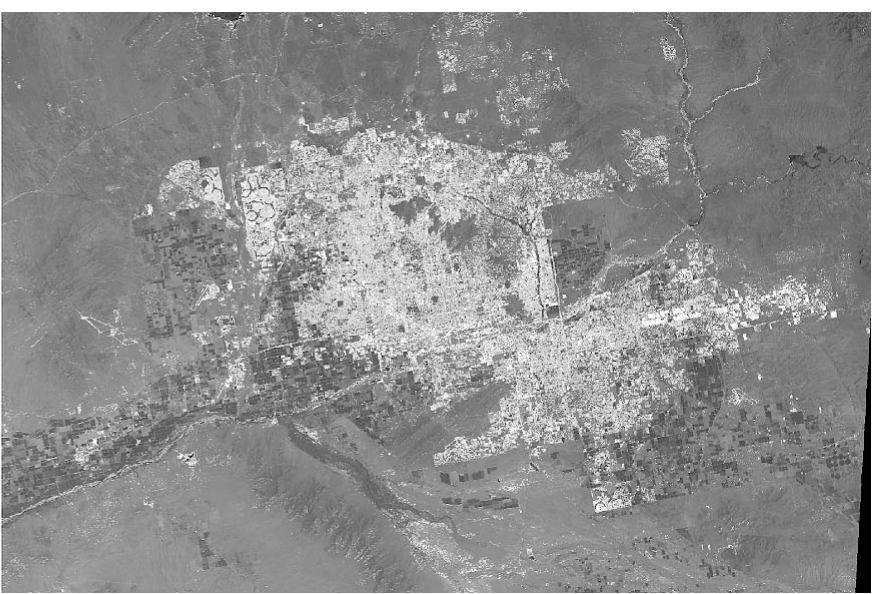

Fig. 1. Phoenix in 1985 (left) and 1998 (right). Bright regions are urbanized areas. The approximate total urbanized areas in 1985 and 1998 are 960 and $1520 \mathrm{~km}^{2}$, respectively. The total size of the area under study is $114 \times 85.5 \mathrm{~km}\left(9747 \mathrm{~km}{ }^{2}\right)$, of which $114 \times 76.0 \mathrm{~km}\left(8664 \mathrm{~km}^{2}\right)$ is visible in the figure 

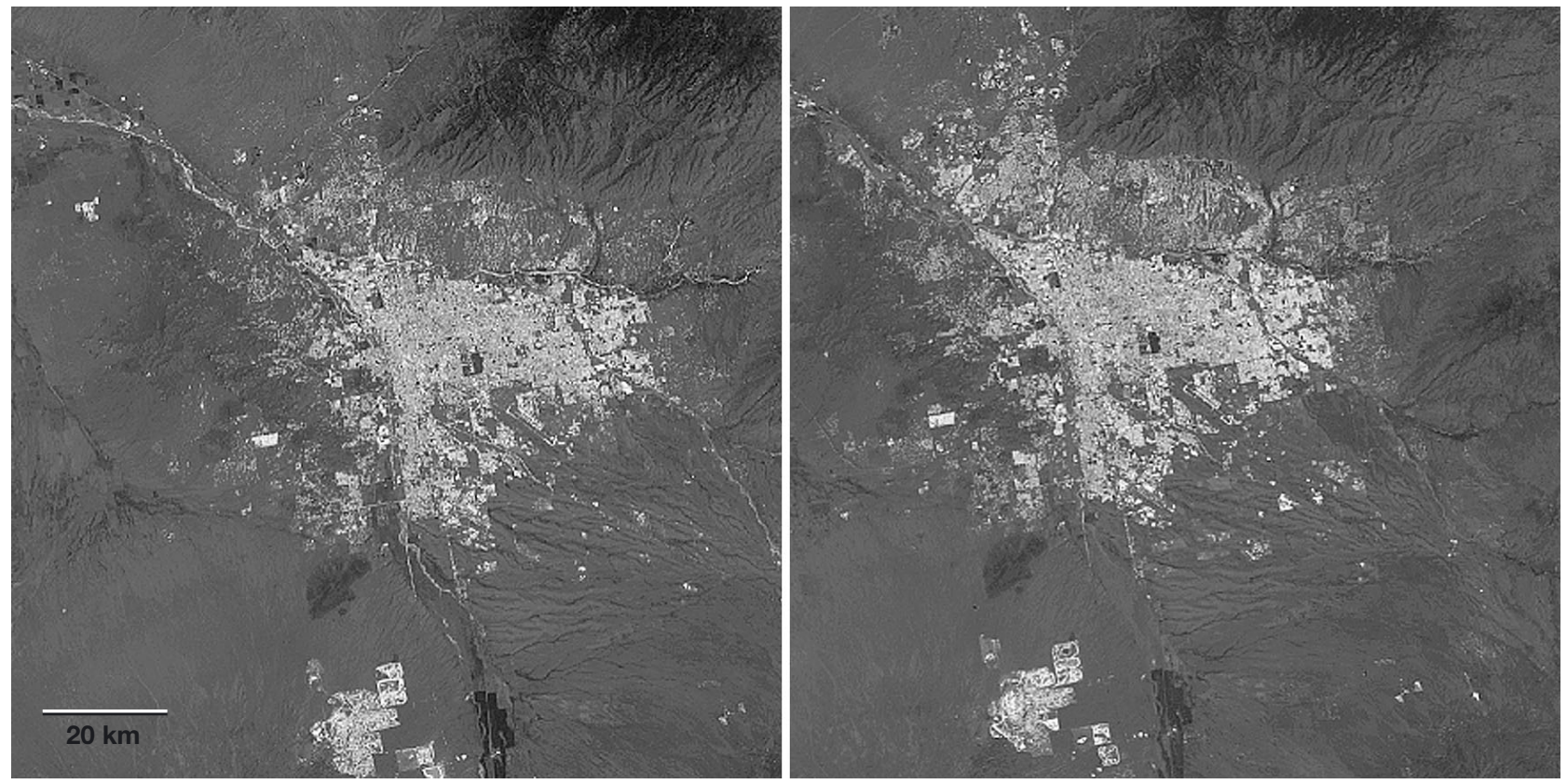

Fig. 2. Tucson in 1984 (left) and 1998 (right). Same type of data as in Fig. 1. The total urbanized areas in 1984 and 1998 are 265 and $335 \mathrm{~km}^{2}$, respectively. The size of the area studied is $57 \times 57 \mathrm{~km}\left(3249 \mathrm{~km}^{2}\right)$, which is completely represented in the figure

Fig. 3 exhibits the result of the image analyses for the urbanized land area, from 1984 to 1999. For Phoenix, the area grew from $960 \mathrm{~km}^{2}$ in 1985 , to $1512 \mathrm{~km}^{2}$ in 1998, representing a $58 \%$ growth over a 14 yr span. This corresponds to an annual average growth of $39 \mathrm{~km}^{2} \mathrm{yr}^{-1}$. For Tucson, the total growth from 1983 to 1997 is $70 \mathrm{~km}^{2}$, which corresponds to an annual average growth of $4.7 \mathrm{~km}^{2} \mathrm{yr}^{-1}$; the magnitude of the urbanized land area is comparatively small $(\sim 1 / 5$ of the Phoenix area). The scatter in the data is due to occasional misclassifications.

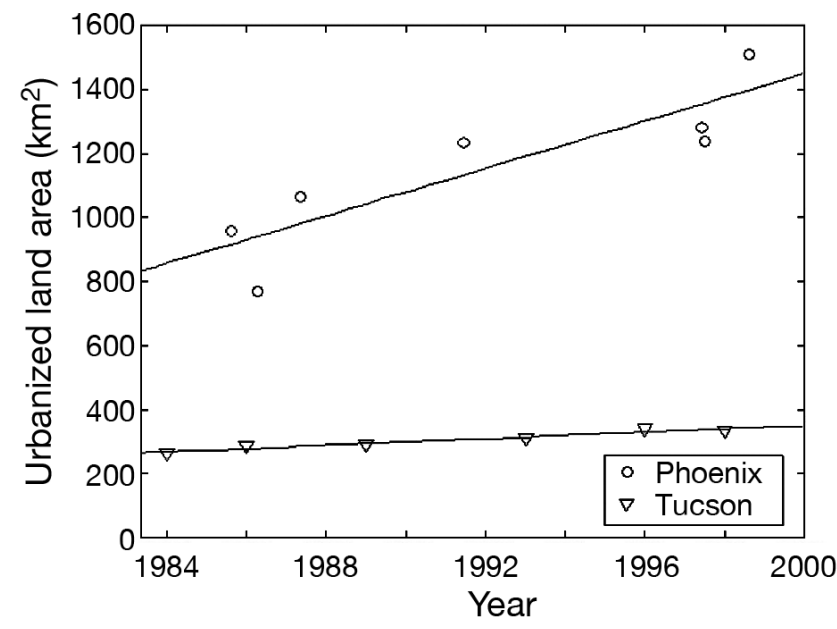

Fig. 3. Urbanized land area of Phoenix and Tucson versus time, as determined by the classification algorithm. Trend lines derived from linear least-squares approximations

\subsection{Temperature records}

Fig. 4 shows the average maximum and minimum temperatures that were recorded at representative sites in both areas. There has been a steady increase in both the maximum and nighttime minimum temperatures for Phoenix, with the increase in the latter being more pronounced. The Tucson maximum temperature, however, indicated a minute level of decrease, while the nighttime minimum exhibits a definite increase. Autocorrelation coefficients for the temperature trends for Phoenix range from 0.83 to 0.57 for time lags of 1 to $5 \mathrm{yr}$, respectively. The autocorrelation coefficient for Tucson is in the same range, at 0.75 for a 1 yr time lag, showing the persistent upward trend along with the urbanized land area.

\subsection{Scaling of the energy balance}

Here we examine the relationship between the urbanized land area and nighttime minimum temperatures by plotting them on the same graph. However, some normalization was required, as we were comparing 2 quantities (temperature and area) with different units. We used the reference area and the nighttime minimum temperature in 1986 for both Phoenix and Tucson, to normalize the data as $A_{\text {norm }}=\left(A-A_{\text {ref }}\right) / A_{\text {ref }}$ and $T_{\text {norm }}=\left(T_{\min }-T_{\text {ref }}\right) / T_{\text {ref. }}$. The normalized urbanized land area and the nighttime minimum temperatures 

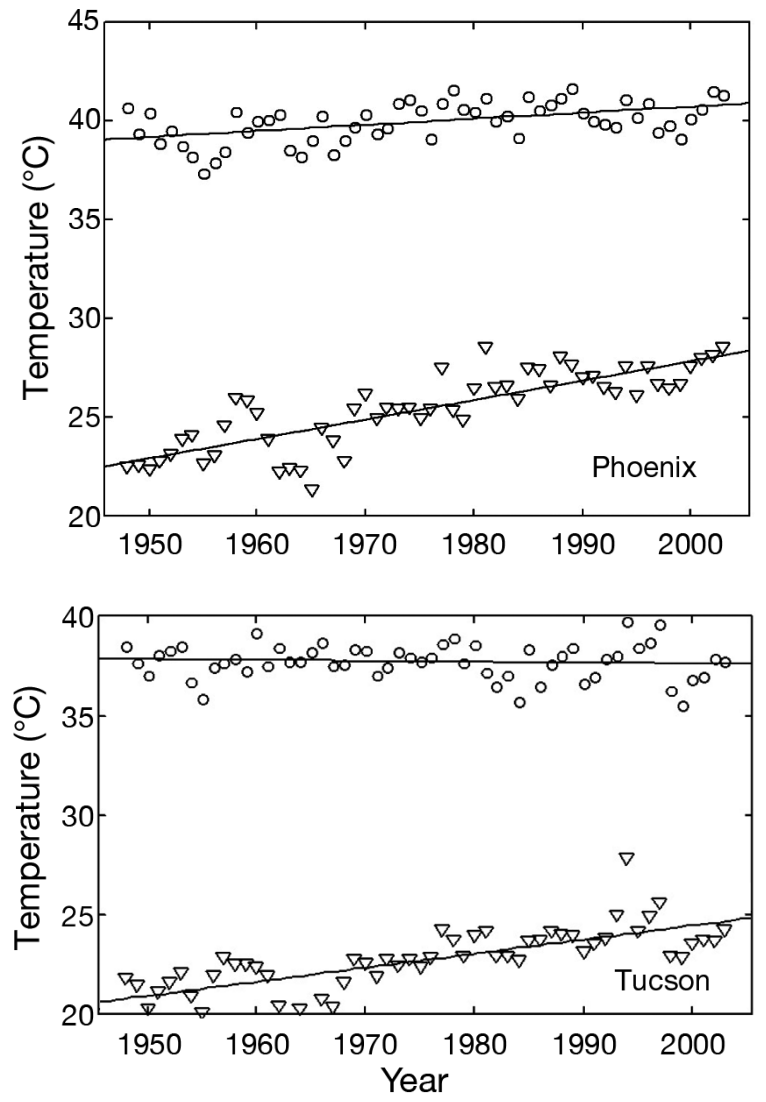

Fig. 4. Average summertime maximum (O) and minimum $(\nabla)$ temperatures measured at Sky Harbor International Airport (Phoenix) and at the University of Arizona (Tucson) from 1948 to 2003 (mean of the average monthly temperatures from June to August). Source: Global Historical Climate Network, see www.ncdc.noaa.gov/oa/climate/climatedata.html\#daily

have steadily increased as a function of time (Fig. 5). Correlation coefficients between the normalized urbanized area and temperature are 0.265 and 0.188 for Phoenix and Tucson, respectively. The data set suggests a scaling where $T_{\text {norm }} \sim A_{\text {norm }}$, or possibly $T_{\min } \sim L$, where $L$ is the length scale of the urbanized land area which is taken to be the square-root of the area.

Kalnay \& Cai (2003) analyzed the temperature record from every NCEP-NCAR station below the $500 \mathrm{~m}$ altitude in the continental United States since 1950, and found land-use change to be the dominant mechanism for the increase in the nighttime minimum temperature. Gallo et al. (1995) also used satellite image data to obtain normalized vegetation indices for 28 cities, and found that this quantity is directly correlated with the increase in the nighttime minimum temperatures. Stabler et al. (2005) concluded that the Phoenix UHI is directly correlated with the growth of the urban land area. A similar study by Chen et al. (2006) indicated a similar correlation between the urban land area and

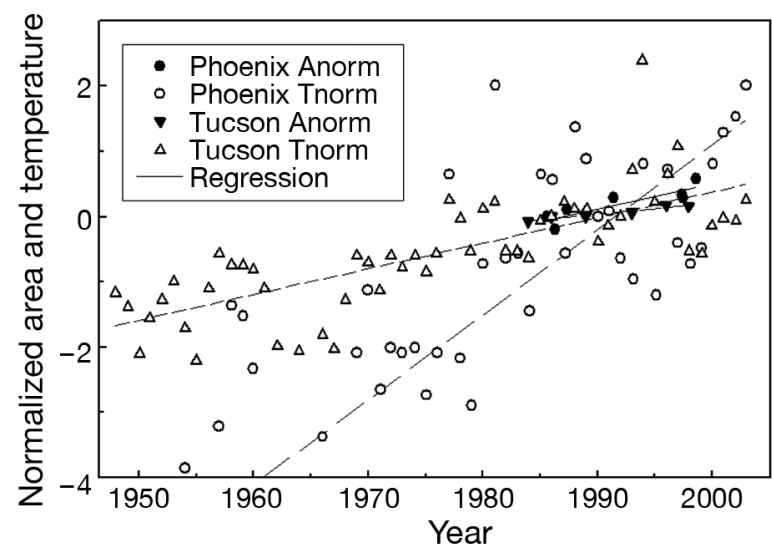

Fig. 5. Long-term trends in the normalized area and temperature, Anorm: normalized area; Tnorm: minimum (nighttime) temperature

nighttime minimum temperature in Shenzhen, China. Thus, there is ample evidence for a strong correlation between the urban land area and the increase in the nighttime minimum temperatures. On the other hand, Oke (1973) and Torok et al. (2001) reported on correlations of the temperature bias as a logarithmic function of the population. The anthropogenic effect of population vs. the urbanized land surface effects (such as convective or radiative heat exchange) are not easy to discern, particularly since the urbanized land area typically increases as the population increases. However, factors such as the domestic output of heat due to air conditioning is quite small compared to the radiative forcing of the urbanized surfaces (roof tops, walls, roads and concrete surfaces). This is because the heat output from air-conditioners mainly offsets the heat that was transferred from the outside into dwellings in the first place.

We can establish a precise relationship between the nighttime minimum temperatures and the length scale of the urban areas by considering the largescale thermal energy transport of urban areas. First, it can be shown that the steady-state heat transfer from horizontal surfaces (the urbanized surfaces that are heated during the day by solar irradiation) has a very weak (if any) dependence on the length scale, for either free or forced convection conditions (see Appendix 1). For a horizontal surface dissipating heat through steady-state natural or wind-assisted convection, the dependence on the length scale of the urban area is only of $L^{0}$ or $L^{-0.2}$, respectively (details are included in Appendix 1). For steady-state natural convection of heat from horizontal surfaces corresponding to 0 wind velocity, there is no length scale dependence $\left(L^{0} \sim\right.$ constant). This is to be expected since heat rises essentially vertically under natural convection conditions, and the horizontal length scale 
does not affect this vertical air motion. For steadystate wind-assisted or forced convection over horizontal surfaces ( $L^{-0.2}$ dependence), the length scale would have to increase $3 \times$ (or the area $9 \times$ ) for the heat loss to decrease by $20 \%$. Again, because the length scale dependence for this mode of heat transfer is very weak at best and therefore fails to explain the current trends, we have given the mathematical analyses in Appendix 1. For typical humidity levels in Phoenix and Tucson, vapor mole fractions at the average minimum temperatures range from 0.013 to 0.017 during July, August, and September. The molecular weights of air and water vapor are 28.97 and $18.02 \mathrm{~kg} \mathrm{kmol}^{-1}$, respectively, while the specific heats of air and water vapor are 1.007 and $1.868 \mathrm{~kg} \mathrm{kmol}^{-1}$. Therefore, the approximate contribution of water vapor to the atmospheric heat content roughly ranges from 0.2 to $1.7 \%$. This means that the effect of the humidity in the air on the heat content, i.e. atmospheric temperature, is typically $1 \%$ or less. Thus, humidity has a large impact on evaporative cooling processes, since the difference in the vapor pressure will drive evaporation. However, humidity at the levels observed in Phoenix and Tuscon has a very small effect on the atmospheric heat content.

Thus, we are led to look at the thermal energy balance of an urban area in a different framework, this time involving the time-dependent thermal energy transport for the 'air mass' above the urban surface that 'includes the temporal change', the advection terms and the surface heat flux $\left(\dot{q}^{\prime \prime}\right)$.

$$
\rho_{\text {air }} C_{\mathrm{p}} \frac{\partial T_{\mathrm{air}}}{\partial t}+\rho_{\mathrm{air}} C_{\mathrm{p}} u \frac{\partial T_{\mathrm{air}}}{\partial x}=\dot{q}^{\prime \prime}
$$

where $T_{\text {air }}=$ temperature of the air mass above the urban land surface, $\rho_{\text {air }}=$ air density, $c_{\mathrm{p}}=$ specific heat of air, $u=$ convection velocity in the horizontal direction, and $\dot{q}^{\prime \prime}=$ heat flux from the land surface. Eq. (3) is a classical conservation of energy equation involving the air mass above the urban surface (Kays \& Crawford 1993). The first term on the left-hand side represents the rate of change of thermal energy (heat) contained in that air mass, which can change due to the remaining terms in the equation. Adding heat (the source term on the right-hand side) increases the heat content, while the convection or advection term (the second term on the left-hand side) diminishes the heat content. If we substitute the appropriate time and length scales for the time-dependent and advection terms, we get

$$
\frac{\Delta T_{\mathrm{air}}}{\Delta t} \approx-U_{\infty} \frac{T_{\mathrm{air}}-T_{\infty}}{L}+\dot{q}^{\prime \prime} / \rho c_{\mathrm{p}}
$$

where $\Delta T_{\text {air }}$ is the temperature change for the air mass over the urban area, $U_{\infty}$ is the average advection velocity (e.g. wind speed), $T_{\infty}$ is the temperature of the air outside the urban area, and $L$ is the length scale of the urban area. Here, we can see that the advective transport (which is now moved to the first term on the right-hand side of Eq. 4) decreases as $L$ increases, thereby reducing the temperature change (cooling) over the urban area, reflecting the data trend in Fig. 6. The surface heat flux $\dot{q}^{\prime \prime}$ has little or no length scale dependence (again see Appendix 1), and can therefore be omitted from any further scaling considerations involving the length or area. It should be noted that the heat flux, by definition, is the heat loss rate per unit area. A surface that is heated to a certain temperature during the day would be dissipating heat by a rate that is mainly determined by the temperature difference between the surface and the air mass. The physical picture that arises is that solar irradiation heats up the urban surfaces (ground, roof tops, etc.) during the day. During nighttime, the heat from the urban surface $\left(\dot{q}^{\prime \prime}\right)$ forms a high-temperature air mass which needs to be convectively cooled by surrounding air. As the urban scale increases as in Eq. (4), the rate of cooling decreases as the length scale $L$ increases, resulting in higher nighttime temperatures. Eq. (4) does not include the latent heat or heat content of the water vapor (humidity), effects of which are small as noted above; however, this term can be added in the energy balance if the humidity effect becomes important.

We can establish a more precise relationship between the temperature and the length scale change by performing a basic dimensional analysis on Eq. (3) after dropping the heat flux term, again since the heat flux term does not have any length scale dependence. Nondimensionalizing the variables in Eq. (3) with $u^{*}=$ $u / U_{\infty}, T^{*}=T / T_{\infty}, t^{*}=t / \tau$, and $x^{*}=x / L$, we find the following relationship:

$$
-\frac{\partial T^{*}{ }_{\text {air }}}{\partial t^{*}}=\frac{\tau U_{\infty}}{L} \frac{\partial T^{*}{ }_{\text {iir }}}{\partial x^{*}}
$$

For the temporal rate term on the left-hand side of Eq. (5), we can write its dependence as

$$
-\frac{\partial T_{\text {air }}^{*}}{\partial t^{*}}=-\frac{\frac{T_{\text {air }}-T_{\text {ref }}}{T_{\text {ref }}}}{\frac{t}{\tau}} \sim-\frac{T_{\text {air }}-T_{\text {ref }}}{T_{\text {ref }}} \equiv-\tilde{T}
$$

In Eq. (6), we are considering the temperature at some fixed time $t / \tau$, and defining the ratio of the temperature departure to the reference temperature as $\widetilde{T}$. The nondimensional temperature gradient on the right-hand side can similarly be estimated using

$$
\frac{\partial T_{\text {air }}^{*}}{\partial x^{*}}=\frac{\frac{T_{\text {air }}-T_{\infty}}{T_{\infty}}}{\frac{L}{L_{\text {ref }}}} \sim \frac{L_{\text {ref }}}{L}
$$


It should be noted that the temperature difference in Eq. (7) is between the air mass above the urban area and the far-field surrounding area, which would be a constant under fixed weather conditions.

The terms on the right-hand sides of Eqs. (6) and (7) are plotted in Fig. 6, with linear regressions fitted through each data set. To make a direct comparison, we plotted $1-\tilde{T}$ and $L / L_{\text {ref }}$ so that both numbers start from 1 at the reference year (1986). Moreover, we multiplied $\tilde{T}$ by a proportional constant, c, to reflect the multiplicative factor of $L / \tau U_{\infty}$ in Eq. (5). The above equations (Eqs. 5, 6 \& 7) show that there should be a linear dependence on the nondimensional temperature change and the inverse of the length scale. Moreover, the proportionality constant $C$ between them is given by $L / \tau U_{\infty}$ in Eq. (5). Taking $\tau$ $=300 \mathrm{~s}$ for the time scale for the temperature change during nighttime, a length scale of $30000 \mathrm{~m}$ (as in the case of Phoenix) and $U_{\infty} \sim 1 \mathrm{~m} \mathrm{~s}^{-1}$, we find the order of magnitude for the proportionality constant $\mathrm{C}$ to be 100 . The length scale $L$ is the scale taken from the analysis of the satellite images, the time scale of temperature change taken as $5 \mathrm{~min}=300 \mathrm{~s}$, and the advection velocity is in the general range of the meteorologically observed wind speeds. With these numbers, we find proportionality constants of 160 and 80 for the Phoenix and Tucson data in Fig. 6, respectively, with the ratio being 2 . This ratio is in very good agreement with the ratio of the length scales of Phoenix and Tucson, which are 1.82 in 1986 and 2.11 in 1998, i.e. Phoenix has an equivalent diameter that is $\sim 2 \times$ that of Tucson, or an area that is

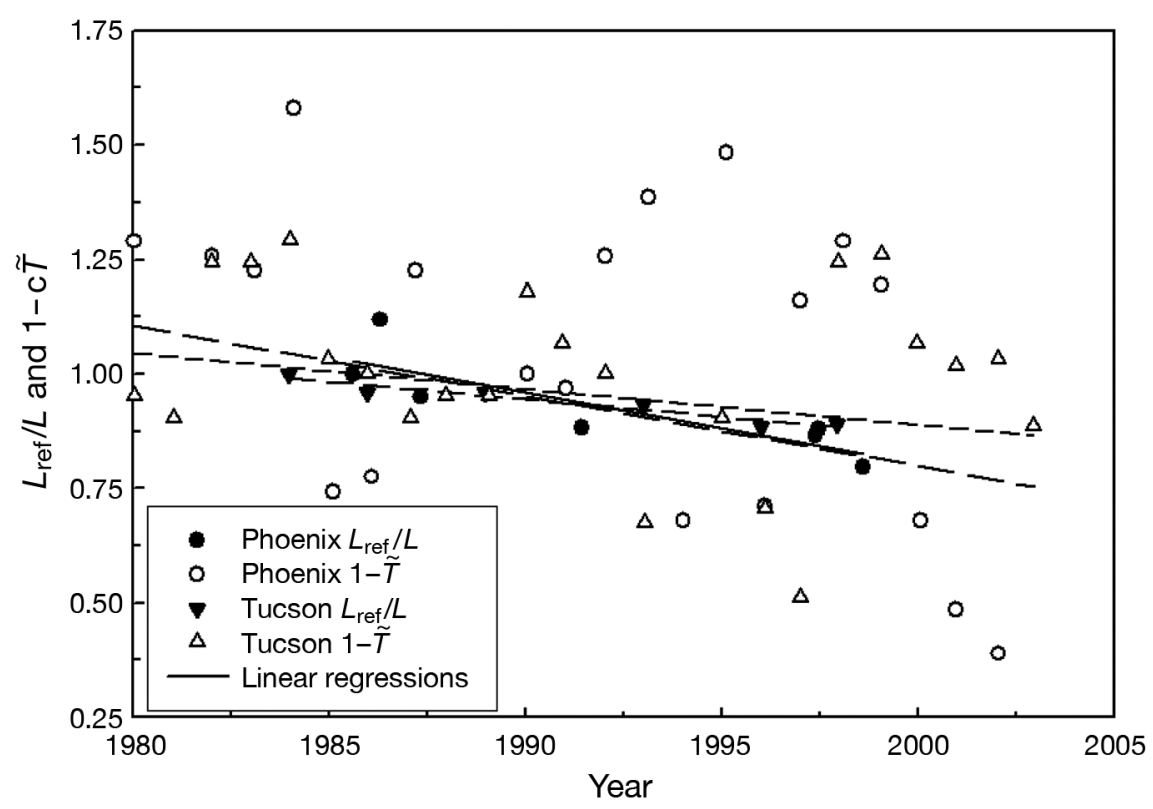

Fig. 6. $L_{\mathrm{ref}} / L$ and $1-c \tilde{T}$ for Phoenix and Tucson from 1980 to 2003. $C$ is the proportionality constant used in transforming Eq. (5) $\sim 4 \times$ that of Tucson, and this is reflected in the above proportionality constant. There is scatter in both the temperature and image-derived surface area data; nonetheless, the trend is definitely observable and is consistent with the above scaling analysis. Further work to examine several other cities in the US for this scaling relationship is the subject of a follow-up work from this study. In summary, the normalized nighttime minimum temperature and the inverse of the normalized length scale should have a linear dependence on the proportionality constant that scales with the length scale of the urban area. Both of these aspects were observed in the data from both Phoenix and Tucson-2 cities with disparate length scales but similar meteorological conditions.

\section{CONCLUSIONS}

The nighttime minimum temperature, which is an indicator of the UHI effect, was seen to follow the length scale of the urbanized land area in the data for Phoenix and Tucson that were examined in this study. Using the time-dependent thermal energy balance equation for the air mass above the urbanized area, this relationship can be explained by the rate of cooling of the air mass over the urban region, which scales with the advection rate. This rate in turn is inversely proportional to the length scale of the urbanized land surface. Viewed in this perspective, the atmospheric advection of heat is an important factor in determining the severity of the UHI effects, as the rate of advection diminishes when the length scale of the urban area is increased. The departure of the nighttime minimum temperature from the normal level should thus scale as the square-root of the urbanized area, with other conditions being similar. In addition, the scaling factor, i.e. the slope of the above relationship, agrees with estimates based on the city sizes of Phoenix and Tucson. A further extension of this scaling to other cities with varying boundary conditions would require incorporation of other parameters, such as the local average temperature, humidity, wind conditions and surface geometrical and radiative properties. Such a scaling, if also proven to be accurate, will obviously be of value in estimating the UHI effects for arbitrary groups of cities. 


\section{LITERATURE CITED}

Atkinson BW (2003) Numerical modelling of urban heat island intensity. Boundary-Layer Meteorol 109:285-310

Bacci P, Maugeri M (1992) The urban heat island of Milan. Il Nuovo Cimento C 15:417-424

Baker LA, Brazel AJ, Selover N, Martin C and others (2002) Urbanization and warming of Phoenix (Arizona, USA): impacts, feedbacks and mitigation. Urban Ecosyst 6: 183-203

Brazel AJ, Selover N, Vose R, Heisler G (2000) The tale of two climates: Baltimore and Phoenix urban LTER sites. Clim Res 15:123-135

Brazel AJ, Gober P, Lee S, Grossman-Clarke S, Zehnder J, Hedquist B, Comparri E (2007) Determinants of changes in the regional urban heat island in metropolitan Phoenix (Arizona, USA) between 1990 and 2004. Clim Res 33: 171-182

Chen XL, Zhao HM, Li PX, Yin ZY (2006) Remote sensing image-based analysis of the relationship between urban heat island and land use/cover changes. Remote Sens Environ 104:133-146

Chow SD (1992) The urban climate of Shanghai. Atmos Environ 26B:9-15

Christy JR, Norris WB, Redmond K, Gallo KP (2006) Methodology and results of calculating Central California surface temperature trends: evidence of human-induced climate change? J Clim 19:548-563

> De Laat ATJ, Maurellis AN (2006) Evidence for influence of anthropogenic surface processes on lower tropospheric and surface temperature trends. Int J Climatol 26:897-913

Di Sabatino S, Leo LS, Hedquist BC, Carter W, Fernando HJS (2009) Results from the Phoenix UHI Experiment. Geophys Res Abstr 11:EGU2009-EGU12778

Emmanuel R, Fernando HJS (2007) Urban heat islands in humid and arid climates: role of urban form and thermal properties in Colombo, Sri Lanka and Phoenix, USA. Clim Res 34:241-251

Gallo KP, Tarpley JD, McNab AL, Karl TR (1995) Assessment of urban heat islands: a satellite perspective. Atmos Res $37: 37-43$

Ho A (2005) Analyses of satellite-based data to examine environmental effects of urbanization. BSc thesis, Arizona State University

Holman JP (2009) Heat transfer, 10th edn. McGraw Hill, p 725

- Kalnay E, Cai M (2003) Impact of urbanization and land-use change on climate. Nature 423:528-531

Kays WM, Crawford (1993) Convective heat and mass transfer, 3rd edn. McGraw Hill, p 480

Landsberg HE (1975) Atmospheric changes in a growing community. Urban Ecol 4:53-81

$>$ Oke TR (1973) City size and the urban heat island. Atmos Environ 7:769-779

Park HS (1986) Features of the heat island in Seoul and its surrounding cities. Atmos Environ 20:1859-1866

Pearlmutter D, Berliner P (1998) Street canyon geometry and microclimate: designing for urban comfort under arid conditions. In: Maldonado E, Yannas S (eds) Environmentally friendly cities. Proc PLEAS '98, Lisbon, Portugal, p 165-166

Rex TR (2000) Population density in metropolitan Phoenix. Report on Brookings Growth Case Study, Morrison Institute for Public Policy, Phoenix, AZ

Rydin Y (1992) Environmental dimensions of residential development and the implications for local planning practice. J Environ Plann Manage 35:43-61

Stabler LB, Martin CA, Brazel AJ (2005) Microclimates in a desert city were related to land use and vegetation index. Urban For Urban Green 3:137-147

Stone B Jr, Rogers MO (2001) Urban form and thermal efficiency: how the design of cities influences the urban heat island effect. J Am Plann Assoc 67:186-198

Torok S, Morris C, Skinner C, Plummer N (2001) Urban heat island features of southeast Australian towns. Aust Meteorol Mag 50:1-13

Yague C, Zurita E, Martinez A (1991) Statistical analysis of the Madrid urban heat island. Atmos Environ 13: $880-890$

Appendix 1. Scaling for the steady-state thermal energy transport from horizontal surfaces

For steady-state free convection from upward-facing horizontal surfaces, the normalized heat transfer coefficient, or the Nusselt number, is given by Holman (2009) as

$$
N u=\frac{h L}{k}=C(G r \operatorname{Pr})^{1 / 3}
$$

where $\mathrm{Nu}$ is the Nusselt number, $h$ is the heat transfer coefficient, $L$ is the length scale, $k$ is the thermal conductivity, $G r$ is the Grashoff number and $\mathrm{Pr}$ is the Prandtl number. The constant $C$ is in the order of 0.1 and depends on the magnitude of the Grashoff number. The Grashoff number in turn is defined as

$$
G r=\frac{g \beta\left(T_{s}-T_{\infty}\right) L^{3}}{V^{2}}
$$

where $g$ is the gravitational acceleration, $\beta$ is the volumetric coefficient of expansion, and $v$ is the kinematic viscosity. For urban areas, the Grashoff number is in the order of $10^{22}$. Due to the 1/3rd power for Gr in Eq. (A1), the Nusselt num- ber has a linear dependence on the length scale $L$. However, when converted to the heat transfer coefficient $h$, the scaling for free convection from the horizontal surface is $h$ constant. Thus, this scaling fails to explain the trends observed in Figs. 6 \& 7.

What about the steady-state forced convection due to atmospheric winds? A typical correlation for turbulent forced convection over a surface has the following scaling:

$$
N u=\frac{h L}{k}=\operatorname{Pr}^{1 / 3}\left(A \operatorname{Re}^{0.8}-B\right)
$$

where $A$ and $B$ again are constants and Re is the Reynolds number based on the length scale.

$$
\mathrm{Nu}=\frac{U_{\infty} L}{\mathrm{~V}}
$$

Hence, using Eqs. (A1) \& (A2), we arrive at $\mathrm{h} \sim 1 / \mathrm{L}^{0.2}$, which is a very weak dependence and again does not explain the trends contained in Figs. 6 \& 7 . 